\title{
Reducing Loads on Buried Pipes by Lightweight Backfilling Materials
}

\author{
Antonio Miglio \\ Hydraulic and Pipeline Consultant Engineer \\ Italy
}

Analysis of buried pipes shows that loads have a significant influence on the sizing of these types of buried structures, especially for pipes buried under high fills. The use of lightweight backfilling materials (LBMs) can be highly beneficial to the buried pipes since - thanks to their lower density - they are able to reduce the loads on the pipe, both immediately and in the long term, and, therefore, pipe stresses and deflections, allowing savings in the design of pipe wall thickness. Furthermore, the use of lightweight backfilling materials offers significant savings in time, cost and quality compared to the use of natural fill materials.

Lightweight materials can consist of a variety of materials, both natural and/or derived from industrial process (e.g., expanded polystyrene blocks (EPS - geofoam); cellular concrete (foamed concrete); lightweight aggregate like expanded clay aggregate; foam glass aggregates; recycled tire chips mixed with soil). Thanks to their own mechanical and physical properties, lightweight backfilling materials are rapidly gaining acceptance and application in the installation of buried structures. Therefore, the comprehension of their mechanical behavior and the interaction with the buried pipes are fundamental to evaluate correctly the loads, and, therefore, perform an optimum and safe design of these types of buried structures.

The keynote lecture will provide an in-depth overview of lightweight backfilling materials used in the installation of buried pipes, showing how these materials are able to reduce the loads on the pipe.

Special attention will be focused on EPS Geofoam, a lightweight material widely used in the installation of buried pipes, especially when pipes are buried under high fills.

Moreover, instructions will be given on when and how to use a specific lightweight material for pipe embedment and backfill. Information regarding limitations of these materials will be discussed.

In addition, the keynote lecture will analyze: a) the evaluation of the loads when lightweight backfilling materials are used; and b) the procedure to design a buried pipe when a lightweight backfilling material is used.

Finally, case studies regarding the load reduction on buried pipes by lightweight backfilling materials will be shown. 